\title{
Algorithms for lattice QCD: progress and challenges
}

\author{
Stefan Schaefer \\ CERN, Physics Department, CH-1211 Geneva 23, Switzerland \\ Humboldt Universität zu Berlin, Institut für Physik, Newtonstr. 15, 12489 Berlin, Germany
}

\begin{abstract}
The development of improved algorithms for QCD on the lattice has enabled us to do calculations at small quark masses and get control over the chiral extrapolation. Also finer lattices have become possible, however, a severe slowing down associated with the topology of the gauge fields has been observed. This may prevent simulations of lattices fine enough for controlling the continuum extrapolation. This conference contribution introduces the basic concepts behind contemporary lattice algorithms, the current knowledge about their slowing down towards the continuum and its consequences for future lattice simulations.

CERN-PH-TH/2010-274, SFB/CPP-10-120
\end{abstract}

Keywords: lattice QCD algorithms, critical slowing down, Wilson fermions PACS: $12.38 . \mathrm{Gc}, 05.10 . \mathrm{Ln}$

\section{INTRODUCTION}

Lattice QCD has witnessed a dramatic progress during the last decade. Whereas simulations ten years ago where still carried out either in the quenched approximation or at sea quark masses far above the physical values, we can now calculate with much lighter quarks with masses down to the physical values of the up and down quark. Only part of this progress is due to increased computer resources. The larger part comes from improved algorithms, in particular the way the fermion determinant, responsible for the effects of the sea quarks, is included in the simulations. The ideas behind this progress will be reviewed in the first part of this contribution.

By their nature, lattice calculations are never done "at the physical point", i.e. in continuum space-time, infinite volume and with six dynamical quark flavors at precisely the physical masses, ideally taking the effect of the full standard model into account. In particular, a lattice simulation will always be at a finite lattice spacing and the result at several fine lattice spacings $a$ has then to be extrapolated to $a=0$. The systematic error associated with this extrapolation depends on the ability to simulate at lattice spacings which are much smaller than the scales involved in the problem. For the physics of light quarks $\Lambda_{\mathrm{QCD}}$ is the relevant scale. For relativistic heavy quarks, however, additionally the scale set by the quark's mass plays an important role and the lattice spacing $a$ has to be sufficiently small, i.e. $a m_{q} \ll 1$. For the charm quark, e.g., one therefore needs lattice spacings well below $0.05 \mathrm{fm}$ for precision results.

In the generation of the ensembles fine enough to control the continuum extrapolation, however, a grave problem occurs: some modes in the simulation move increasingly slowly, in particular the phenomenon is visible in the topological charge of the gauge configuration. The simulation rarely tunnels between topological sectors.

Some critical slowing down is expected in any simulation as critical points are approached, here the continuum limit. A typical rate of this increase is with the second power of the correlation length. In gauge theory, however, we find a critical exponent of $z \approx 5$ instead of two, making simulations of fine lattices practically impossible. The details of these statements are covered in the second half of this writeup. The writeup finishes with an overview of the current state of the lattice simulations with Wilson fermions. The status of staggered quark simulations has been covered by Gottlieb at this conference[1].

\section{DYNAMICAL FERMIONS}

Because of their anti-commuting nature, there is no natural way to treat fermions in numerical simulations. The textbook version of the path integral for QCD is unfortunately not suitable for numerical simulations since it contains integrals over Grassmann variables. They can be performed analytically, which introduces the determinant of the Dirac operator, with the remaining integrals over the gauge degrees of freedom $U$

$$
Z=\int[d U] \prod_{f}^{N_{f}} \operatorname{det}\left[D\left(m_{f}\right)\right] \exp \left(-S_{g}[U]\right)
$$

with $S_{g}$ the gauge action and $D\left(m_{f}\right)$ the lattice version of the Dirac operator with quark mass $m_{f}$. Since $D$ is a large matrix, computing its determinant is virtually impossible and it is replaced by a path integral over bosonic "pseudo-fermion" fields $\phi$

$$
\operatorname{det} D^{2}\left(m_{f}\right) \propto \int[d \phi]\left[d \phi^{\dagger}\right] \exp \left(-\phi^{\dagger} \frac{1}{D^{\dagger} D} \phi\right),
$$


which works for even numbers of degenerate flavors, because the matrix in the exponent has to be Hermitian positive definite. Although applying this identity seems innocent, using it in a straight forward manner simulating the resulting path integral (containing gauge and pseudo-fermion fields) with the Hybrid Monte Carlo (HMC) algorithm[2] turns out to be unfeasible for light quarks and fine lattices. This was famously summarized by Ukawa at the Lattice conference in 2001[3], where he gave as the cost of generating a decent sized ensemble for two-flavors of dynamical Wilson quarks as

$$
C\left[\frac{n_{\text {conf }}}{1000}\right]\left[\frac{m_{\pi} / m_{\rho}}{0.6}\right]^{-6}\left[\frac{L}{3 \mathrm{fm}}\right]^{5}\left[\frac{0.1 \mathrm{fm}}{a}\right]^{7}
$$

with $C=2.8$ Tflops years. At the physical value of the pion mass, where $m_{\pi} / m_{\rho} \approx 0.17$, this would be impossible even with today's machines. Just physically light quarks, for which $3 \mathrm{fm}$ are too small, would require a peta-flops machine for several years despite the coarse lattice spacing of $0.1 \mathrm{fm}$.

There are two, related, basic insights which lead to the progress of the last decade. The first was that the estimate of the determinant, which is provided by one realization of the pseudo-fermion field $\phi$, is not good enough. Better estimators have to be used, but in a way, which is meaningful from the physics point of view and which also can be introduced into lattice QCD algorithms.

The second insight, which often also provides a solution to the first, is that the ultra-violet and the infra-red physics of the theory are different and also need to be treated differently. By separating the two, one can deal with them according to their requirements. But the successful methods to achieve this splitting also provide improved estimators of the fermion determinant.

The initial break-through into this direction is by Hasenbusch with his mass preconditioning [4, 5], where first, the fermion matrix is split into two parts

$$
\operatorname{det} D\left(m_{f}\right)=\operatorname{det} D(M) \operatorname{det}\left[D\left(m_{f}\right) D^{-1}(M)\right]
$$

with $M$ a mass larger than $m_{f}$. The first term is therefore dominated by the UV physics, whereas the second part is largely infrared. On each of the two terms, Eq. 2 is applied and the resulting partition function can be simulated with the standard HMC algorithm, yielding a dramatic speed-up, for the right choice of $M$, of course.

Another successful split-up of the determinant is domain decomposition (giving the DD-HMC its name), which was introduced by Lüscher in Ref. [6], see Fig. 1 for an illustration. Here, the lattice is decomposed into blocks and the Dirac operator is split into one living only inside the blocks and a correction term, which accounts for the rest

$$
\operatorname{det} D\left(m_{f}\right)=\prod_{\text {blocks }} \operatorname{det} D_{\text {block }}\left(m_{f}\right) \cdot \operatorname{det} R
$$

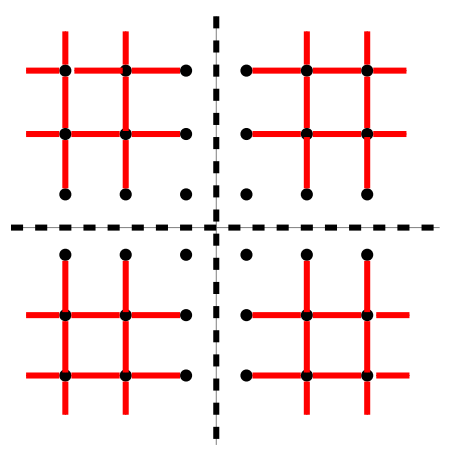

FIGURE 1. In the DD-HMC algorithm, the lattice is split into blocks, thereby separating the UV from the IR part of the fermion action.

This provides an obvious geometric separation in ultraviolet and infra-red part with the corresponding speed-up in the algorithm.

And finally, the third way of splitting the determinant leading to the RHMC[7] is to use

$$
\operatorname{det} D=\prod_{i=1}^{N} \operatorname{det} D^{1 / N}
$$

The separation between IR and UV is less clear, however, it has the advantage that the fermion flavors do not have to come in even numbers of degenerate quarks. It is therefore the method of choice for simulating the strange and charm quark, using the identity $\operatorname{det} D=\operatorname{det} \sqrt{D^{\dagger} D}$.

The algorithms profit from the separation of the UV and the IR because these two contributions evolve on very different time scales. The gauge field has much faster fluctuations than most of the UV of the fermions, which in turn evolve much faster than the infra-red. This makes the use of specialized methods possible [8, 9, 10], which move the modes on separate time scales.

For example, just the block decomposition of the DDHMC algorithm leads to a cost formula of

$$
C\left[\frac{n_{\text {conf }}}{1000}\right]\left[\frac{20 \mathrm{MeV}}{m}\right]\left[\frac{L}{3 \mathrm{fm}}\right]^{5}\left[\frac{0.1 \mathrm{fm}}{a}\right]^{6},
$$

with $C=0.5$ Tflops years and $m$ the running $\overline{M S}$ sea quark mass at $2 \mathrm{GeV}$ [11]. To compare with Eq. 3, we note that to leading order in the chiral expansion $m_{\pi}^{2} \propto m$. Thus the exponent governing the cost of going chiral, which was 6 ten years ago, has dropped to 2. Equally important, the overall normalization is reduced by a factor of 100. With mass preconditioning, similar performance can be reached[12].

To summarize, of the three algorithms, the RHMC follows the most the idea of an improved estimator of the fermion determinant. It provides a splitting of the determinant into equal parts, reducing the fluctuations 
introduced by the pseudo-fermions. The separation of IR and UV, however, is not as obvious, but both profit from the improved estimation.

At the other end, the block decomposition of DDHMC is clearly designed for the separation of the UV from the IR. Since the action is split into the two parts, whose relative size can be tuned by the size of the blocks, also a better estimator is provided.

The mass preconditioning takes an intermediate position. The IR/UV separation is softer, since in both factors of Eq. 4 contain both parts of the spectrum, however, with different weights. But it also provides a clear handle on getting a better estimator for the fermion determinant. The identity of Eq. 4 can be iterated and with a larger number of suitably chosen masses $M_{i}$, a systematically better estimate can be reached.

\section{Solver}

All evidence speaks very much in favor of the efficiency of the simple observation that the infra-red is different from the ultra-violet part of the physics and that respecting this physics can lead to this very beneficial effects. The art is to bring it in a feasible and cost-efficient way to the numerical simulations.

It has long been known that the infra-red part of the Dirac operator is responsible for the high cost of solving the Dirac equation, which is the most expensive part of lattice simulations. In some special applications, therefore, the low eigenvectors are removed, leading to a dramatic speed-up, however, at the cost of computing these eigenvectors, whose number grows with the volume. Lüscher realized the dominant contribution to this space can be constructed from localized modes[13] in a very cheap way. Basically, very few approximate eigenvectors of the Dirac operator are spatially cut apart and then recombined in all possible ways. This gives then the major part of the low eigenspace up to some physical energy. The achievement of this method is that its cost just grows linearly with the volume, instead of previous methods, which had at least a $V^{2}$ scaling.

For the solution of the Dirac equation, removing the space constructed from these local modes virtually eliminates all increase in the cost of this operation as the quarks get lighter, i.e. the factor of $m^{-1}$ in Eq. 5. At moderately light quark masses, gains of a factor of 10 have been observed. These savings have been demonstrated in the original DD-HMC setup[14] as well as in mass preconditioned HMC[12]. A very similar idea is the adaptive multi-grid[15].

It remains to be noted, that the idea of treating the infra-red part of the Dirac operator's spectrum special has not stopped here. Also for observables, computing the contribution from the infra-red more precisely than the UV part has proven to be very beneficial[16, 17].

\section{APPROACHING THE CONTINUUM}

The advances in the fermionic sectors have led to significant optimism and large ensembles of gauge configurations at different sea quark masses and lattice spacings have been produced. However, going to finer lattices, a severe slowing down of the simulations has been observed[18, 19]. This is not new phenomenon and had been observed previously in particular in pure gauge theory, however, with dynamical fermions the problem is slightly less severe.

\section{Background}

In Markov Chain Monte Carlo simulations, an algorithm is a probabilistic procedure to generate a sequence of field configurations $U_{i}$

$$
U_{1} \rightarrow U_{2} \rightarrow U_{3} \rightarrow \cdots \rightarrow U_{N}
$$

given by a transition probability $T\left(U^{\prime} \leftarrow U\right)$. Under certain conditions, in particular stability

$$
P(U)=\sum_{U^{\prime}} P\left(U^{\prime}\right) T\left(U \leftarrow U^{\prime}\right)
$$

the $U_{i}$ are then distributed according to a given probability distribution $P$. Because of this process, the probability distribution of $U_{i+1}$ depends on $U_{i}$, which leads to correlations among subsequent measurements of observables $A_{i}=A\left(U_{i}\right)$. These are described by the auto-correlation function

$$
\Gamma_{A}(t)=\left\langle\left(A_{i}-\langle A\rangle\right)\left(A_{i+t}-\langle A\rangle\right)\right\rangle
$$

and in an even more concise way by the integral, the auto-correlation time

$$
\tau_{\mathrm{int}}(A)=\frac{1}{2}+\sum_{i=1}^{\infty} \frac{\Gamma_{A}(t)}{\Gamma_{A}(0)} .
$$

The error $\sigma_{A}$ for an estimate from $N$ subsequent measurements is then given by

$$
\sigma_{A}=\frac{\sqrt{\operatorname{var}(A)}}{\sqrt{N / 2 \tau_{\mathrm{int}}}} .
$$

This is the ordinary error formula, which effectively differs from the one without correlation just by the reduction of the number of measurements $N$ by a factor of $2 \tau_{\text {int }}$. 


\section{Critical slowing down}

The cost of a simulation is thus proportional to $\tau_{\text {int }}$ and in the continuum limit — approaching a continuous phase transition-one expects it to grow with a power-law

$$
\tau_{\text {int }} \propto a^{-z}
$$

with $a$ the lattice spacing and $z$ the dynamical critical exponent. The whole phenomenon is called critical slowing down and can be viewed in analogy to static critical phenomena and their scaling laws. However, it has to be stressed that the value of $z$ does not only depend on the properties of the underlying theory, but also on the algorithm with which it is simulated. For some spin models, algorithms with $z \approx 0$ have been found, like cluster or multi-grid algorithms, however, for Yang-Mills theory or QCD, such an algorithm does not exist. For a generic small step algorithm, one expects $z \approx 2$, based on the idea that information is distributed in a random walk and needs to spread over a correlation length (here proportional to $a^{-1}$ ) to give an independent measurement. The cost formulae in Eqs. 3 and 5 seem to assume $z \approx 1$.

The cost of an independent measurement is proportional to the cost of a single update times the number of updates needed. A single update will most certainly have a cost proportional to $a^{-4}$ for fixed volume in four dimensions. For Hybrid Monte Carlo, $a^{-5}$ is a typical behavior. Combined with the effect of critical slowing down, this gives $a^{-5-z}$, which can give a very sizeable effect for a large $z$.

\section{Hybrid Monte Carlo}

As described above, virtually all current simulations are using a variant of the HMC algorithm. It is therefore pivotal to know the behavior of their cost when the continuum limit is approached. Although this cost will depend on the observable in question, a safe simulation needs to be in a situation where all observables decorrelate much faster than the full statistics. This is necessary, because the modes, which are being moved by the transition matrix $T$, couple to all observables-barring some symmetry explicitly prohibiting the coupling. The situation needs to be such that one is able to detect from the simulation itself the coupling of slowly moving modes to the observables in question, which requires that we see sufficient movement in all possible quantities.

In order to determine the dynamical critical exponents of lattice simulations, we performed a pure gauge study with the Wilson gauge action using the same algorithms as used for QCD simulations, mainly DD-HMC, but we also tested the behavior of pure HMC $[19,20]$. The main result is displayed in Fig. 2. It shows how the integrated

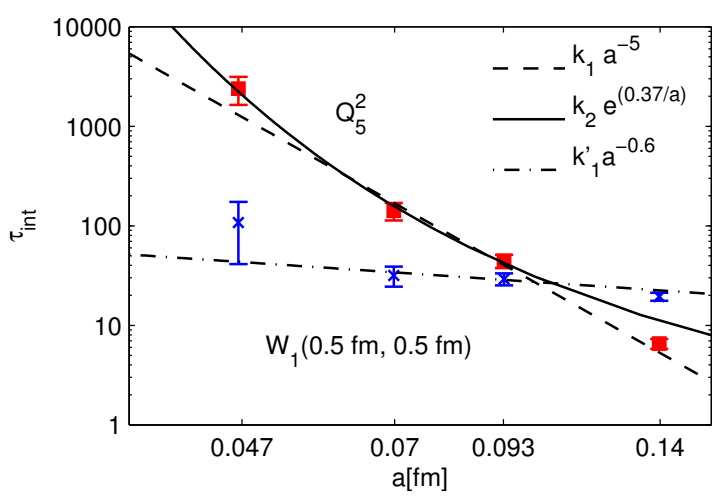

FIGURE 2. Integrated auto-correlation time vs. the lattice spacing. $Q^{2}$ is the topological charge squared, whose behavior is compatible with $z \approx 5, W$ is the square Wilson loop which for which $z \approx 1$.

auto-correlation time rises as the continuum limit is approached. This behavior is different for different observables. We display the topological charge, for which a very steep rise compatible with a dynamical critical exponent of $z \approx 5$. However, an exponential behaviorfor which evidence is presented in [21] — cannot be excluded. The picture is very different for the (smeared) square Wilson loop of size $0.5 \mathrm{fm}$, which we show because it turns out to be the loop with the slowest evolution. The rise is compatible with $z \approx 1$, actually less severe than a simple random walk picture suggests. This is already an indication that a decoupling between the slow modes governing the topological charge and some other observables occurs. The problem is that in principle, one has to check observable by observable, whether the situation is under control, or whether the slow modes contribute significantly.

In Fig. 3, we make a comparison between the autocorrelation behavior of pure gauge theory and of dynamical two-flavor simulations with Wilson quarks at roughly the same lattice spacing. It is striking that the auto-correlation functions are virtually identical between the two set-ups for all observables at which we looked, including meson masses, quark masses, decay constants and the plaquette. Only in the topological charge a significant difference appears. The same phenomenon can also be observed in pure gauge theory, when moving to a different gauge action. We tested it for the Iwasaki gauge action and also found the only difference compared to the Wilson gauge action in the auto-correlations of the topological modes.

The slowing down of the topological charge does not come as a complete surprise. It has long been part of the folklore of the field that in the continuum there is a separation between the topological charge sectors. From the point of view of the lattice, however, the emergence 

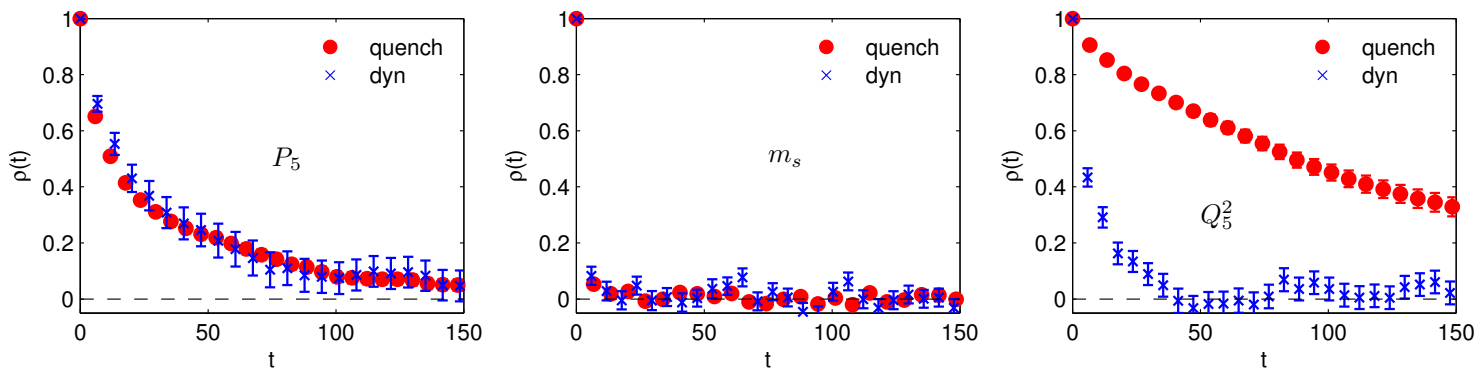

FIGURE 3. Comparison between the normalized auto-correlation function $\rho\left(t=\Gamma(t) / \Gamma(0)\right.$ for pure gauge and dynamical $N_{f}=2$ simulations. On the left, we show the smeared plaquette, then in the center the quark mass corresponding to the strange quark, on the right the square of the topological charge.

of these sectors has long been unclear. A typical gauge configuration is very rough and contains a large number of small objects (dislocations) which carry topological charge. This makes a separation in charge sectors of the connected field space seem arbitrary.

Recently, Lüscher[22] proposed a new definition of the charge using the gradient flow on the field space, smoothing the fields in a well defined way. He could show that the configurations, to which a certain charge cannot be attributed unambiguously, die out very quickly as the lattice spacing is reduced with a power of roughly $a^{-6}$. It is clear, that a small step algorithm has problem with this kind of situation: since the algorithm is supposed to do importance sampling, it will stay during the evolution in the region of important configurations; steps bridging a less important region are by construction not included. If the configurations between sectors are rapidly becoming less important, the transition from one topological sector to the other will obviously be suppressed accordingly.

\section{STATUS OF WILSON FERMION SIMULATIONS}

The ability to simulate QCD at light fermion masses and small lattice spacings has put many collaborations into the position to generate ensembles on which interesting physics can be studied. An overview of the status of the simulations with Wilson fermions can be found in Tab. 1. All collaborations use formulations, in which leading lattice effects are removed. CLS[23, 24] and PACS-CS[25] both use non-perturbative improvement, ETMC [26, 27] uses twisted mass fermions at maximal twist which profit from automatic $\mathscr{O}(a)$ improvement. The BMW collaboration[28] uses tree-level improved Wilson fermions which couple through "HEX" smeared links to the gauge field. The QCDSF and UKQCD collaborations also use gauge field smoothing in their nonperturbatively improved SLiNC fermions[29]. All col- laborations have produced data sets with light sea quarks. The problems described in the previous section limit current studies to about $0.05 \mathrm{fm}$, and even there, worries about slow modes are in place. There are two major obstacles for even lighter fermions these days: finite volume effects and instabilities or possible lattice phase transitions.

The effects of finite volume can easily be kept small by a lattice size much larger than the pion wave length $L \gg m_{\pi}^{-1}$. However, larger $L$ still comes with the fifth power in all cost formulae. Cutting the pion mass by half therefore requires roughly a factor of 30 in computer time, just from this criterion alone.

The second obstacle are instabilities or even phase transitions as the quark mass is lowered. Since this writeup is about Wilson quarks, which explicitly break chiral symmetry, the latter is a real possibility (e.g. the Aoki phase discussed in Ref. [30]). Instabilities in the simulations occurring at small quark mass have been discussed[31], which come from the fact that the spectrum of the Wilson operator, because of the lack of chiral symmetry, is not bounded from below by the quark mass.

Therefore, all types of Wilson fermions can only reach a finite minimal quark mass for a given lattice spacing. The finer the lattice, the smaller this minimal mass. How fine a lattice one needs to simulate the light quarks at their physical parameters depends on the action. The way this problem is mitigated in current set-ups used by the PACS-CS[25] or the BMW[28] collaboration is to use a special gauge action or smeared fermion action, respectively, which suppress the dislocations, thought to be the cause of the problem. These collaborations therefore could report simulations at the physical value of the light quark masses.

\section{SUMMARY}

Lattice computations have gone a long way. Light quarks are light and their effect is taken into account in the 
TABLE 1. Status of current Wilson fermion simulations: $N_{f}$ is the number of sea quark flavors, $a$ the lattice spacing and $m_{\pi}$ the minimal sea pion mass.

\begin{tabular}{cccll}
\hline collaboration & fermion action & $N_{f}$ & $a[\mathrm{fm}]$ & $m_{\pi}[\mathrm{MeV}]$ \\
\hline BMW & tl HEX Wilson & $2+1$ & $0.05 \ldots 0.12$ & $120 \ldots$ \\
CLS & NP imp. Wilson & 2 & $0.05 \ldots 0.09$ & $250 \ldots$ \\
ETMC & tw. Wilson & 2 & $0.05 \ldots 0.1$ & $280 \ldots$ \\
& & $2+1+1$ & $0.08 \ldots 0.09$ & $270 \ldots$ \\
PACS-CS & NP imp. Wilson & $2+1$ & 0.09 & $135 \ldots$ \\
\hline
\end{tabular}

vacuum. However, they are still expensive. In particular, decreasing the lattice spacing comes with a significant critical slowing down of observables like the topological charge. The measured $z \approx 5$ means that the total cost of simulations rises like $a^{-10}$. This requires either very sizable computing resources or a better algorithm which moves these fields more efficiently. Such an algorithm has not been found yet. Certainly, the phenomenon casts doubt on whether the statistical errors in current simulations are truly under control and, with it, the extrapolation to the continuum limit which is so crucial for final answers.

Given the decoupling between the modes which are prominent in the topological charge and responsible for its slow evolution and, e.g. the Wilson loop apparent in Fig. 2, one might wonder whether it matters at all. In particular in a large volume, the global topological charge should have very little effect on any reasonably local observable. However, from a general Monte Carlo perspective, we can determine the expectation values observables, their fluctuations and the associated autocorrelations only from the simulation itself. Since we now know of auto-correlations of the size of a typical total statistics, we have to worry about even longer, undetected ones. To gain confidence in any Markov Chain Monte Carlo, it is necessary that all correlations are much smaller than the total length of the chain. To believe that only the topological charge is affected by the increase in auto-correlations just seems naive.

In the end, for the continuum extrapolation, the lattice simulations are at a similar point now as they were ten years ago for the chiral extrapolation. Back then, simulating pions with less then $500 \mathrm{MeV}$ seemed impossible, with costs exploding with the sixth power. Now we are facing a similar exponent for the increasing autocorrelations towards the continuum. But the solution of the problem of the chiral limit gives hope that also the continuum extrapolation will find a solution in the not too distant future.

Acknowledgements. It is a pleasure to thank M. Hasenbusch, M. Marinkovic, R. Sommer and F. Virotta for many interesting discussions and fruitful collaboration on various subjects which contributed to this talk. This work has been supported in part by the
DFG through the SFB TR/9.

\section{REFERENCES}

1. S. Gottlieb (2010), these proceedings.

2. S. Duane, A. D. Kennedy, B. J. Pendleton, and D. Roweth, Phys. Lett. B195, 216 (1987).

3. A. Ukawa, Nucl.Phys.Proc.Suppl. 106, 195-196 (2002).

4. M. Hasenbusch, Phys. Lett. B519, 177-182 (2001).

5. M. Hasenbusch, and K. Jansen, Nucl. Phys. B659, 299-320 (2003).

6. M. Lüscher, Comput. Phys. Commun. 165, 199 (2005).

7. M. Clark, and A. Kennedy, Phys.Rev.Lett. 98, 051601 (2007).

8. J. C. Sexton, and D. H. Weingarten, Nuclear Physics B 380, 665 - 677 (1992).

9. A. Ali Khan, et al., Nucl.Phys.Proc.Suppl. 129, 853-855 (2004).

10. C. Urbach, K. Jansen, A. Shindler, and U. Wenger, Comput. Phys. Commun. 174, 87-98 (2006).

11. L. Del Debbio, L. Giusti, M. Lüscher, R. Petronzio, and N. Tantalo, JHEP 02, 056 (2007).

12. M. Marinkovic, and S. Schaefer, arXiv: 1011.0811.

13. M. Lüscher, JHEP 07, 081 (2007).

14. M. Lüscher, JHEP 12, 011 (2007).

15. J. Osborn, et. al., arXiv: 1011.2775.

16. T. A. DeGrand, and S. Schaefer, Comput.Phys.Commun. 159, 185-191 (2004).

17. L. Giusti, P. Hernandez, M. Laine, P. Weisz, and H. Wittig, JHEP 0404, 013 (2004).

18. S. Schaefer, R. Sommer, and F. Virotta, PoS LAT2009, 032 (2009).

19. S. Schaefer, R. Sommer, and F. Virotta, arXiv:1009.5228.

20. S. Schaefer, and F. Virotta, arXiv: 1011.5151.

21. L. Del Debbio, G. M. Manca, and E. Vicari, Phys.Lett. B594, 315-323 (2004).

22. M. Lüscher, JHEP 1008, 071 (2010).

23. G. von Hippel, R. Sommer, J. Heitger, S. Schaefer, and N. Tantalo, PoS LATTICE2008, 227 (2008).

24. B. Brandt, et al., arXiv: 1010.2390.

25. S. Aoki, et al., Phys.Rev. D81, 074503 (2010).

26. P. Boucaud, et al., Comput.Phys.Commun. 179, 695-715 (2008).

27. R. Baron, et al., JHEP 1006, 111 (2010).

28. S. Durr, et al., arXiv: 1011.2403.

29. W. Bietenholz et al., PoS LAT2009 (2009) 102.

30. S. Aoki, Phys. Rev. D 30, 2653-2663 (1984).

31. L. Del Debbio, L. Giusti, M. Lüscher, R. Petronzio, and N. Tantalo, JHEP 0602, 011 (2006). 